\title{
"Ensamblaje" de macroinvertebrados acuáticos y su relación con variables fisicoquímicas en un río de montaña en Colombia
}

\author{
Duberly Mosquera-Restrepo ${ }^{1 *} \&$ Enrique Javier Peña-Salamanca ${ }^{1}$ \\ Grupo de Investigación en Biología de Plantas y Microorganismos, Universidad del Valle, Calle 13 \# 100 - 00 Cali, \\ Colombia; duberlymosquera@unicauca.edu.co, enrique.pena@correounivalle.edu.co \\ * Correspondencia
}

Recibido 02-V-2019. Corregido 11-IX-2019. Aceptado 17-IX-2019.

\begin{abstract}
Macroinvertebrate "assemblage" and its relationship with physicochemical variables in a mountain stream in Valle del Cauca, Colombia. Because of the influence of different human activities, rivers and their associated biodiversity are under stress. The aim of this study was to evaluate the diversity of aquatic macroinvertebrates of the Jordán River and its relation to water quality. Mining, water harvesting, livestock, agriculture, recreation and illegal occupation of the territory; take place in the evaluated section (5 $650 \mathrm{~m})$. A sampling site was established at 1173 m.a.s.l. (E1), here the river receives wastewater. The second, at 1069 m.a.s.l. (E2) was set up before the intake of an aqueduct. And the third, at 1019 m.a.s.l. (E3) after the inlet pipe. Concentrations of physicochemical variables $(n=15)$ were measured. Aquatic macroinvertebrates were collected in gravel, pebbles and macrophytes using Surber nets $\left(0.36 \mathrm{~m}^{2}, 500 \mu \mathrm{m}\right)$, a screen net $\left(1.6 \mathrm{~m}^{2}, 350 \mu \mathrm{m}\right)$ and a D-net $\left(0.9 \mathrm{~m}^{2}, 500 \mu \mathrm{m}\right)$. The assemblage structure was assessed using abundance categories. The diversity of aquatic macroinvertebrate and physicochemical variables were associated using canonical correspondence analysis (CCA). The physicochemical variables showed that E3 was the most contaminated site. There were 9 orders, 23 families and 31 taxa. E3 had the lowest richness and in E2 Rhagovelia was dominant. The CCA showed that variables that have the greatest influence on the diversity of macroinvertebrates are oxygen, $\mathrm{BOD}_{5}$, Total Dissolved Solids (TDS) and turbidity. It is concluded that the diversity of macroinvertebrates decreases with increasing organic matter possibly due to sewage and flow decrease.
\end{abstract}

Key words: water quality, biochemical oxygen demand, Elmidae, aquatic insects, Jordán River.

Mosquera-Restrepo, D., \& Peña-Salamanca, E. J. (2019). "Ensamblaje” de macroinvertebrados acuáticos y su relación con variables fisicoquímicas en un río de montaña en Colombia. Revista de Biología Tropical, 67(6), 1235-1246.

Los macroinvertebrados bentónicos son conocidos por ser sensibles a las características de los hábitat (Beauger, Lair, Reyes-Marchant, \& Peiry, 2006) y responder rápido a los cambios en la calidad del agua (Beauger et al., 2006; Klemm, Lewis, Fulk, \& Lazorchak, 1990; Richards, Haro, Johnson, \& Host, 1997; Rosengerg \& Resh, 1993). Pese a que existen estudios sobre la estructura y respuesta de los macroinvertebrados acuáticos a la contaminación en ríos de montaña en diferentes regiones como Polonia (Wyzga et al., 2014),
Alemania (Januschke, Jähnig, Lorenz, \& Herin, 2014), España central (Camargo, Alonso, \& de la Puente, 2005) y Argentina (Fernández, Romero, Peralta, \& Grosso, 2001); en la región neotropical, pese a tener la mayor riqueza taxonómica, existe poco conocimiento del efecto de ciertos contaminantes sobre la diversidad de los macroinvertebrados (Thorp \& Rogers, 2015; Covich, 1988).

La distribución y abundancia de los insectos acuáticos (el principal componente taxonómico del ensamblaje de macroinvertebrados 
acuáticos) frecuentemente son el objetivo de los estudios sobre poblaciones y comunidades acuáticas, asimismo, es importante la determinación e identificación de algunas especies o grupos taxonómicos clave dentro de toda una comunidad (Sánchez-Vélez \& García-Núñez, 1999).

Existe poca información sobre la fauna de ríos, especialmente de macroinvertebrados (Robinson, Tockner, \& Ward, 2002) y si bien en el departamento del Valle del Cauca se han realizado varios estudios (citas) sobre la diversidad de macroinvertebrados de ríos andinos no existe información sobre la composición y estructura de los ensamblajes de macroinvertebrados acuáticos y su relación con las variables fisicoquímicas de calidad de agua en la subcuenca del río Jordán.

La subcuenca del río Jordán (afluente del río Jamundí en el departamento del Valle del Cauca), presenta diferentes actividades que deterioran la calidad de sus aguas como minería (explotación de bauxita y carbón), agricultura y derivación de agua para consumo humano (bocatoma de la planta de tratamiento de agua potable) (Alcaldía de Jamundí, 2007) y presencia de asentamientos humanos con vertimientos de agua residual sin tratamiento alguno (Funvivir, 2008). Estas actividades impactan de manera negativa sobre el río Jordán disminuyendo su caudal y aumentando la concentración de metales, nutrientes y materia orgánica.

El de este estudio fue evaluar la diversidad de macroinvertebrados acuáticos y su correlación con las variables fisicoquímicas asociadas a la calidad del agua en la cuenca media y baja del río Jordán.

\section{MATERIALES Y MÉTODOS}

Área de estudio: El río Jordán está ubicado en el municipio de Jamundí, departamento del Valle del Cauca (Fig. 1). Tiene una longitud de $11.61 \mathrm{~km}$.

El material parental y geológico de la subcuenca del río Jordán corresponde a materiales transportados y depositados por la corriente de los ríos (CVC, DAGMA, \& Parques Nacionales Naturales de Colombia, 2007). El comportamiento del caudal medio mensual para el río Jordán muestra que los meses con caudales bajos corresponde a los periodos de
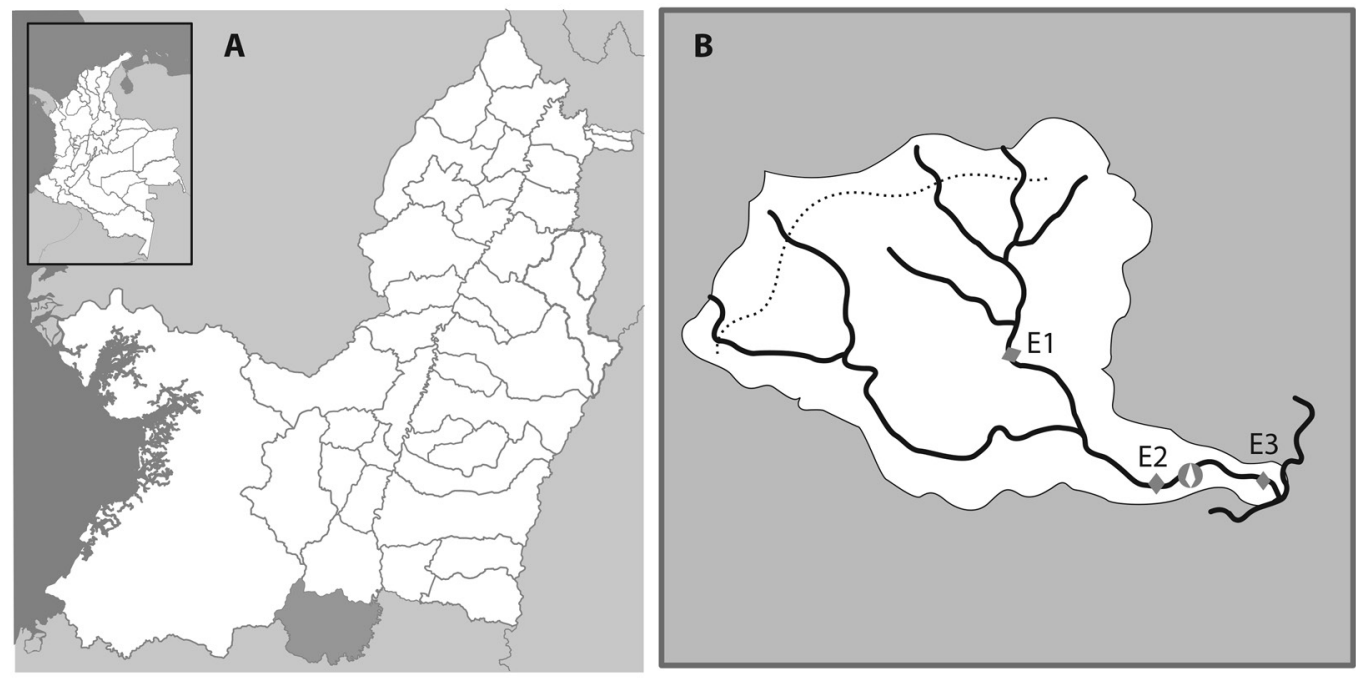

Fig. 1 A. Municipio de Jamundí en el Valle del Cauca (gris oscuro); B. subcuenca del río Jordán mostrando estaciones de muestreo. Los rombos negros ubican las estaciones de muestreo (E), el símbolo de diamante blanco representa la ubicación de la bocatoma del acueducto del municipio de Jamundí. La línea punteada representa el límite del Parque Nacional Natural Farallones de Cali. 
enero - marzo y julio - septiembre (periodos de baja precipitación). Mientras que los meses con caudales altos comprende los periodos de abril - junio y octubre - diciembre (periodos de alta precipitación).

En la subcuenca del río Jordán la precipitación media anual es de $2355 \mathrm{~mm}$, presentando un clima semicálido (CVC, 2009) con pérdida de regulación hídrica debido a los cambios en la cobertura vegetal, y a los procesos extractivos de material forestal de la cuenca, usado para las bocaminas o para la venta en los municipios de Jamundí y Cali (Corporación Autónoma Regional del Valle del Cauca - CVC, DAGMA, \& Parques Nacionales Naturales de Colombia, 2007).

En la parte media y baja del río Jordán se presentan actividades mineras de bauxita, carbón y agregados pétreos (Funvivir, 2008). También se presentan tensores como agricultura, ganadería, actividades recreativas, asentamientos de desarrollo incompleto y captaciones de agua para potabilización (Funvivir, 2008; Alcaldía de Jamundí, 2007; Corporación Autónoma Regional del Valle del Cauca et al., 2007).

El tramo de río estudiado tiene aproximadamente $5650 \mathrm{~m}$ de longitud, iniciando en la cabecera del corregimiento de La Estrella y finalizando en la afluencia del río Jordán en río Jamundí. Los muestreos se realizaron en tres estaciones; se ubicó una estación de muestreo (E1) a $1173 \mathrm{~m}$ de altitud (316'19"' $\left.\mathrm{N} \& 76^{\circ} 36^{\prime} 56^{\prime \prime} \mathrm{O}\right)$, con vertimientos de agua residual doméstica. La segunda estación (E2) se ubicó a $1069 \mathrm{~m}$ de altitud (314'46" N \& $\left.76^{\circ} 36^{\prime} 56^{\prime \prime} \mathrm{O}\right)$ antes de la bocatoma de la planta de tratamiento de agua potable (PTAP). La tercera estación de muestreo (E3), se ubicó a $1019 \mathrm{~m}$ de altitud $\left(3^{\circ} 14^{\prime} 41^{\prime \prime} \mathrm{N} \& 76^{\circ} 35^{\prime} 3^{\prime \prime}\right.$ O), después de la bocatoma y otras fuentes de estrés u otros estresores como la minería y vertimientos. Estas estaciones de muestreo forman parte de las jornadas de campo realizadas por el grupo de Investigación en Biología de Plantas y Microorganismos de la Universidad del Valle desde el 2010.
Medición de variables fisicoquímicas: Se muestrearon quincenalmente, abarcando el patrón bimodal de lluvias para la zona, las siguientes variables fisicoquímicas y microbiológicas: Temperatura, $\mathrm{pH}$, turbiedad (FAU), demanda bioquímica de oxígeno $(\mathrm{mg} / \mathrm{l})$, saturación del oxígeno disuelto (\%), concentración de oxígeno $(\mathrm{mg} / \mathrm{l})$, sólidos disueltos totales (SDT) $(\mathrm{mg} / \mathrm{l})$, conductividad eléctrica $(\mu \mathrm{s} / \mathrm{cm})$, fosfatos (mg/l), nitratos (mg/l), coliformes fecales y totales (NMP 100/ml).

Se empleó la base metodológica para la toma y conservación de muestras de agua que se recopila en los Métodos Normalizados para el Análisis de Aguas: Standard Methods APHA-AWWA-WPCF (2005) y los protocolos Rápidos de la Evaluación de la Calidad Ecológica (GUADALMED - PRECE) (JáimezCuellar et al., 2002).

Determinación de la estructura y composición del ensamblaje de macroinvertebrados acuáticos: Durante un año se muestrearon macroinvertebrados acuáticos cada 45 días abarcando el patrón bimodal de lluvias para la zona (el mismo periodo usado para los muestreos fisicoquímicos y microbiológicos). En cada estación de muestreo se seleccionaron diferentes mesohábitat fluviales. Para la selección de los mesohábitat se siguieron las recomendaciones de Jáimez-Cuellar et al. (2002) y se caracterizaron teniendo en cuenta el tipo de sustrato (Rosguen, 1994), la velocidad de corriente (Berg, 1948) y la presencia de materia orgánica.

La recolección y procesamiento de muestras se realizó siguiendo las sugerencias de Roldán-Pérez (2003) y Barbour, Gerritsen, Snyder y Stribling (1999). Los organismos se recolectaron usando redes surber, tipo D y de pantalla, todas con malla de $500 \mu \mathrm{m}$. Con la red Surber se muestreó un área de $0.09 \mathrm{~m}^{2}$ en mesohábitat con presencia de gravas y rocas pequeñas, velocidad de corriente moderada y presencia de materia orgánica en partículas gruesas. Con la red tipo $\mathrm{D}$ se realizó un barrido de $3 \mathrm{~m}$, para un 
área de $0.9 \mathrm{~m}^{2}$, en mesohábitat con presencia de macrófitas. Con la red de pantalla se muestreó un cuadrante de $1 \mathrm{~m}^{2}$ en mesohábitat con presencia de bolos, y velocidad de corriente muy rápida. En total se realizaron muestreos en tres mesohábitat por estación para un área total de muestreo por estación de $1.99 \mathrm{~m}^{2}$.

Los muestreos corresponden a las campañas de muestreo realizadas desde el 2010 por el Grupo de Investigación en Biología de Plantas y Microorganismos (BPM) de la Universidad del Valle. Los organismos fueron depositados en la colección del Museo de Entomología de la Universidad del Valle (No. 077, MUSENUV), bajo los códigos 28867 hasta el 28884 .

Para la identificación taxonómica se usaron las claves taxonómicas de Hamada, Thorp y Rogers (2018), Domínguez y Fernández (2009), Merrit, Cummins y Berg (2008), Silva-Passos, Nessimian y Ferreira (2007), Domínguez, Molineri, Pescador, Hubbard y Nieto (2006), Posada-García y Roldán-Pérez (2003), Chacón y Segnini (1996), Muñoz (1996), De Castellanos (1994) y MachadoCartagena (1989).

La representatividad de los muestreos se evaluó usando los estimadores Chao 1 y ACE, y la ocurrencia de taxones representados por uno y dos individuos (singletons y doubletons respectivamente) (Gotelli \& Colwell, 2011; Villarreal et al., 2006). Los estimadores se calcularon con la aplicación Stimates 8.20 (Colwell, 2006).

La similitud entre los eventos de muestreo se realizó usando el coeficiente de Bray Curtis (BC), previa transformación de los datos a la función $\log _{10}(\mathrm{X}+1)$ mediante el software PRIMER $^{\circledR}$ (Clarke \& Gorley, 2015). Para la determinación de la representatividad numérica de cada taxón en el ensamblaje de macroinvertebrados acuáticos se realiza una nueva propuesta basándose en el criterio de Lobo y Leighton (1986) y una modificación de las categorías de abundancia de Kobayasi y Ando (1975), asignando colores a las categorías de abundancia para una futura realización de mapas de diversidad (Tabla 1).

\section{Relación de los macroinvertebrados acuáticos con las variables fisicoquímicas: Para evaluar la relación entre el ensamblaje de macroinvertebrados, las estaciones de muestreo y las variables fisicoquímicas y microbiológi- cas, se realizó un análisis de correspondencia canónica (ACC) (Palmer, 1993) usando la aplicación PAST 2.16. El pretratamiento inclu- yó la transformación de todos los datos con la función $\log _{10}(\mathrm{X}+1)$.}

\section{RESULTADOS}

Variables fisicoquímicas y microbiológicas: En las estaciones E2 y E3 se observó un aumento de la temperatura, sólidos disueltos totales, conductividad eléctrica y turbidez en relación con la estación E1. En la estación E3 se registró una mayor concentración de SDT respecto a las demás estaciones.

Las variables asociadas al contenido de SDT en el agua mostraron que en la estación E1 se presenta oligotrofía, con valores entre 16.27 y $21.69 \mathrm{mg} / \mathrm{l}$ y de conductividad eléctrica entre 34.9 y $46.1 \mu \mathrm{s} / \mathrm{cm}$. Se registró presencia de coliformes fecales en todos los eventos de muestreo de cada estación, siendo la estación E1 la que presentó valores más altos; este tipo

TABLA 1

Categorías de abundancia

\begin{tabular}{cccc} 
Categoría & Representatividad & Criterio (sobre el número total de individuos) & Color para mapa de diversidad \\
5 & Dominantes & $>50 \%$ & Azul \\
4 & Abundantes & $>$ media aritmética del número total de individuos & Verde \\
3 & Escasas & $>3 \%<$ media aritmética & Amarillo \\
2 & Raras & $1-3 \%$ & Naranja \\
1 & Muy raras & $<1 \%$ & Rojo \\
\hline
\end{tabular}


de contaminación indica que el río Jordán recibe vertimientos domésticos de asentamientos humanos que no cuentan con soluciones de saneamiento básico.

La concentración de oxígeno disuelto fue superior a $7.09 \mathrm{mg} / \mathrm{l}$ en todos los eventos de muestreo, mientras que el $\mathrm{pH}$ tuvo su mayor variación en la estación ubicada aguas abajo de la bocatoma (E3), entre 5.98 y 7.86. El valor más alto de nitrato se presentó en la estación E3 (19.86 $\mathrm{mg} \mathrm{NO}_{3} / \mathrm{l}$ ) y los fosfatos presentaron el valor promedio más alto en la estación E1 (0.343 $\left.\mathrm{mg} \mathrm{P}_{2} \mathrm{O}_{5} / \mathrm{l}\right)$.
Ensamblaje de macroinvertebrados acuáticos del río Jordán: Según los resultados obtenidos con los 3 métodos de muestreo, la diversidad de macroinvertebrados acuáticos estuvo representada por 9 órdenes, 23 familias y 31 taxones (Tabla 2). La familia Elmidae (Coleoptera) fue la mejor representada con 5 géneros. Los estimadores de riqueza esperada mostraron que hubo una aceptable representatividad de los muestreos (Chao $1=79 \%$ y ACE $=80 \%$ ). La curva de los doubletons tiende a descender, siendo este un buen indicador de la eficacia del muestreo.

TABLA 2

Composición y estructura del ensamblaje de macroinvertebrados acuáticos

\begin{tabular}{|c|c|c|c|}
\hline Taxón & La Estrella (E1) & Antes Bocatoma (E2) & Después Bocatoma (E3) \\
\hline Acanthagrion & 1 & 1 & \\
\hline Anacroneuria & 9 & 6 & 4 \\
\hline Anchytarsus & 4 & 3 & \\
\hline Atanatolica & 5 & 6 & 1 \\
\hline Atopsyche & & 2 & \\
\hline Baetodes & 5 & & \\
\hline Camelobaetidius & 4 & & \\
\hline Ceratopogonidae & & 1 & \\
\hline Corydalus & 4 & & \\
\hline Corixidae & & & 1 \\
\hline Cylloepus & 2 & 17 & 6 \\
\hline Ephemeroptera & & & 1 \\
\hline Hexanchorus & 1 & & \\
\hline Heterelmis & 19 & 2 & 1 \\
\hline Libellulinae & 3 & & 2 \\
\hline Leptonema & 18 & 3 & 1 \\
\hline Leptohyphes & 15 & & \\
\hline Limnocoris & 3 & & \\
\hline Macrelmis & 2 & 8 & 1 \\
\hline Nanomis & 1 & & \\
\hline Phanocerus & & & 1 \\
\hline Phyllogomphoides & 1 & 2 & \\
\hline Planariidae & & 1 & \\
\hline Psephenops & 3 & & \\
\hline Rhagovelia & 2 & 137 & \\
\hline Simulium & 4 & 1 & \\
\hline Smicridea & & 7 & \\
\hline Tanypodinae & 4 & 3 & 1 \\
\hline Thraulodes & 11 & 2 & 2 \\
\hline Tipula & 1 & & \\
\hline Xiphocentron & & & 1 \\
\hline
\end{tabular}


Los géneros con mayor distribución en el tramo estudiado fueron: Anacroneuria, Atanatolica, Cylloepus, Heterelmis, Leptonema, Macrelmis, Rhagovelia y Thraulodes, siendo registrados en todas las estaciones. En la estación E1, no hubo taxón dominante, sin embargo, los géneros: Heterelmis, Leptonema, Leptohyphes, Thraulodes y Anacroneuria, fueron clasificados como abundantes (Categoría 4), mientras que Rhagovelia, Cylloepus y Macrelmis, fueron clasificados como géneros raros (Categoría 2) en esta estación. En la estación E2 Rhagovelia fue dominante (Categoría 5), mientras que Smicridea se clasificó como escaso (Categoría 3) (Tabla 2).

La estación E1 presentó la mayor riqueza (23 taxones), con 8 géneros de presencia restringida a esta estación, mientras que la estación E3 presentó la menor riqueza (14 taxones), sin embargo, ningún taxón fue clasificado como dominante. El índice de Bray Curtis muestra que existe una baja similitud entre las estaciones $(<0.53)$, siendo la estación E3 la que más se aleja en terminos de diversidad.
Correspondencia entre las variables fisicoquímicas y los macroinvertebrados acuáticos: El Análisis de Correspondencia Canónica (ACC) explicó el $43.35 \%$ de la variabilidad de los datos en los dos primeros ejes (Fig. 2). Las variables que mejor aportaron al análisis para el primer componente $(23.82 \%)$ fueron el oxígeno disuelto, la $\mathrm{DBO}_{5}$ y los Sólidos Disueltos Totales (SDT), mientras que para el segundo componente $(19.53 \%)$ fue la turbidez.

Se pueden observar 6 grupos con cierto nivel de asociación: 1) Limnocoris y Corydalus se relacionaron positivamente con el oxígeno disuelto, 2) Xiphocentron se asoció al contenido de materia orgánica, 3) Macrelmis, Atopsyche y Cylloepus se relacionaron con la temperatura, 4) Heterelmis, Thraulodes y Acanthagrion se asociaron con la turbidez, 5) Simulium tuvo correspondencia con los Coliformes Fecales y 6) Leptonema, de la familia Hydropsychidae, se asoció con el aumento del $\mathrm{pH}$.

En cuanto a los eventos de muestreo de cada estación, los realizados en la estación E1 (La Estrella) se agrupan mejor con presencia

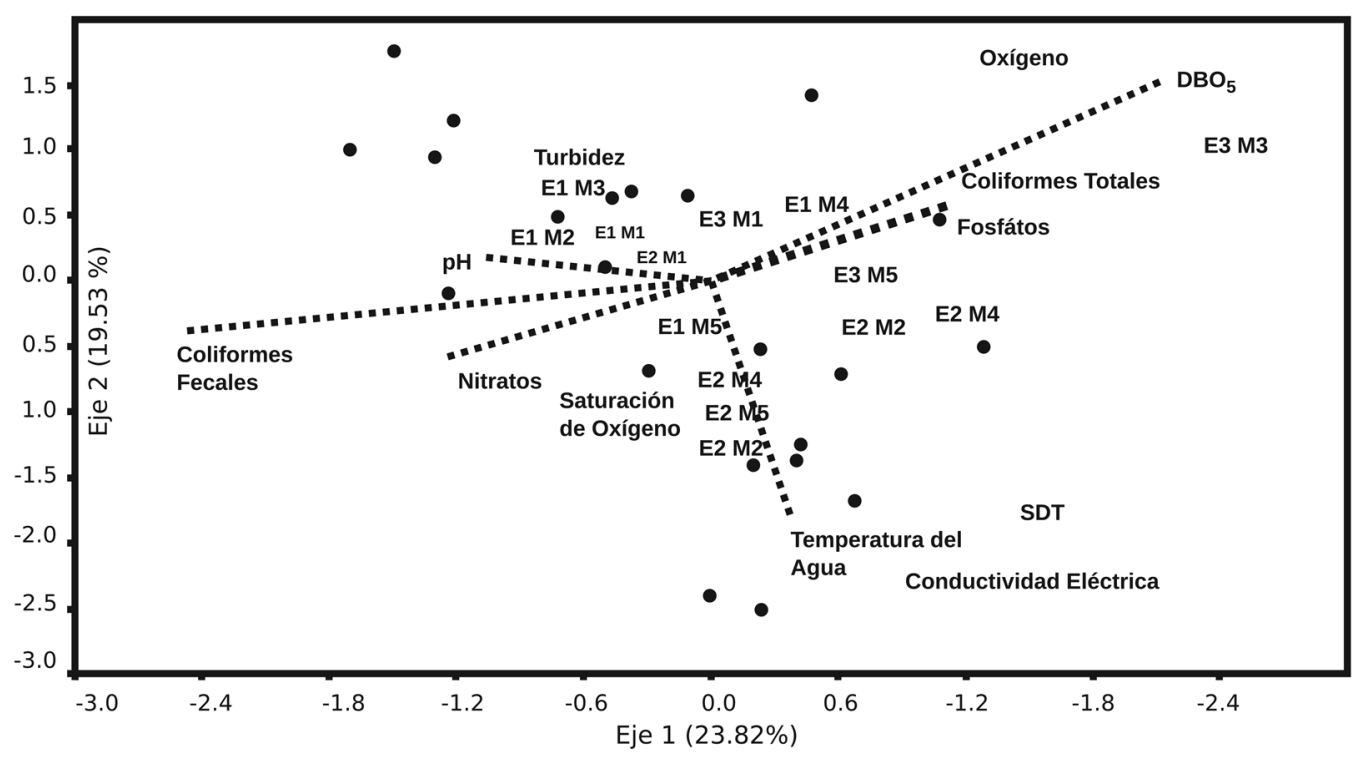

Fig. 2. Diagrama de ordenación del análisis de correspondencia canónica. Los puntos representan las especies. E: Estación de muestreo, M1: Muestreo de febrero, M2: Muestreo de marzo, M3: Muestreo de abril, M4: Muestreo de julio, M5: muestreo de septiembre. 
de géneros sensibles a la contaminación como Anchytarsus, Psephenops y Leptohyphes. Las estaciones E2 (Antes Bocatoma) y E3 (Después Bocatoma) mostraron mayor dinámica entre muestreos principalmente por las variaciones en la abundancia de Rhagovelia.

\section{DISCUSIÓN}

Según Roldán-Pérez (2003), valores de conductividad eléctrica entre 10 y $50 \mu \mathrm{s} / \mathrm{cm}$, son propios de aguas superficiales tropicales de montaña. Las variables asociadas al contenido de sólidos SDT en el agua mostraron que en la estación ubicada a mayor altitud (E1) se presenta una débil mineralización del agua, con valores de SDT ente 16.27 y $21.69 \mathrm{mg} / \mathrm{l}$ y de conductividad eléctrica entre 34.9 y $46.1 \mu \mathrm{s} /$ $\mathrm{cm}$. Esto puede ser debido a la ausencia de una actividad extensiva de ganadería y de minería en esta zona de la subcuenca del río Jordán.

En las estaciones E2 y E3 el aumento de la temperatura, los sólidos disueltos totales, la conductividad eléctrica y la turbidez aumentaron posiblemente debido a la influencia de las actividades productivas y las características propias de cursos de agua más bajos (Castro \& Donato, 2008; Margalef, 1983). En la estación E3, la disminución del caudal por la presencia de la bocatoma pudo generar una mayor concentración de variables de tipo físico como los sólidos. La turbidez en estas estaciones tuvo una variación temporal marcada, posiblemente por la influencia del aumento de los sólidos en suspensión (Roldán-Pérez \& Ramirez-Restrepo, 2008) arrastrados por la escorrentía en los periodos de mayor precipitación.

Según los valores de referencia de diferentes estudios en ecosistemas acuáticos colombianos (Ramírez, Restrepo, \& Viña-Viscaíno, 1997; Roldán-Pérez, 2003; Vásquez-Zapata, 2009), el pH y las variables asociadas a la presencia de materia orgánica y su descomposición, mostraron niveles óptimos para el normal desarrollo de la biota acuática en todas las estaciones.

Los coliformes fecales presentaron niveles altos en todos los eventos de muestreo de cada estación, mostrando que en el tramo estudiado se deben tomar precauciones para el contacto primario (recreación) y tratamiento del agua para su potabilización. Los valores más altos de coliformes fecales se presentaron en la cabecera del corregimiento La Estrella (E1), debido a la presencia de vertimientos de origen doméstico sin tratamiento, y al arrastre de residuos vertidos en la superficie de la subcuenca del río Jordán (Funvivir, 2008; Alcaldía de Jamundí, 2007).

La concentración de oxígeno disuelto fue superior a $7.09 \mathrm{mg} / \mathrm{l}$ en todos los eventos de muestreo, valores considerados óptimos para diferentes actividades como la pesca, recreación y el mantenimiento de flora y fauna según el Decreto No 1594 (1984). El pH tuvo su mayor variación en la estación ubicada aguas abajo de la bocatoma (E3), entre 5.98 y 7.86 . Sin embargo, según Vásquez-Zapata (2009), estos valores se encuentran en el rango de tolerancia de la vida acuática.

Los nutrientes (nitratos y fosfatos) mostraron variación temporal, posiblemente debido a la influencia de los cambios de caudal por el régimen bimodal de lluvias que arrastra material alóctono, y a actividades como la agricultura y vertimientos domésticos de poblaciones legales e ilegales ubicadas desde el corregimiento La Estrella hasta la desembocadura del río Jordán en el río Jamundí. El valor más alto de nitrato se presentó en la estación E3 (19.86 mg $\left.\mathrm{NO}_{3} / 1\right)$ y según Vásquez-Zapata (2009), las concentraciones de nitrato en los sistemas hídricos colombianos con intervención pueden tomar valores hasta de $60 \mathrm{mg} \mathrm{NO}_{3} / 1$. Los fosfatos presentaron el valor promedio más alto en la estación E1 (0.343 mg $\left.\mathrm{P}_{2} \mathrm{O}_{5} / \mathrm{l}\right)$, donde se presentan vertimientos domésticos directos y existe presencia de fincas con actividades pecuarias (Funvivir, 2008).

Anacroneuria se registró donde las variables fisicoquímicas mostraron mejores condiciones de calidad de agua. Esto concuerda con Zúñiga-de-Cardoso (2010), quien al realizar una revisión de la distribución del orden Plecotera en Colombia, argumenta la amplia distribución de Anacroneuria en el país con 
dominancia en la región natural andina. Por otro lado, Bohorquez, Reinoso y Guevara (2011) registran una alta abundancia de Anacroneuria entre los 1000 y 1600 m.s.n.m. en la cuenca del río Prado (Tolima).

Ephemeroptera ocurrió en todas las estaciones con niveles de oxígeno entre 82.2 y $105.8 \%$. Este orden ha sido registrado en ríos del suroccidente colombiano como el Pance (uno de los principales afluentes del río Jamundí) en aguas limpias con niveles de oxígeno entre 91.8 y 103.6 \% (Zuñiga-deCardoso, Rojas-de-Hernández, \& Mosquerade-Aguilera, 1997).

Elmidae es una familia de coleópteros acuáticos verdaderos con distribución cosmopolita, encontrándose principalmente en rápidos de ecosistemas lóticos (Ottoboni-Segura, Valente-Neto, \& Fonseca-Gessner, 2011). Los géneros Cylloepus, Macrelmis y Heterelmis se encontraron en todas las estaciones de muestreo en el río Jordán en mesohabitats de aguas rápidas, sin embargo, la abundancia acumulada de Macrelmis (todos los eventos de muestreo en todas las estaciones) fue menor (11 individuos). Por otro lado, de Hexanchorus sólo se recolectó 1 individuo en la estación E1 y de Phanocerus 1 individuo en la estación E3.

La baja abundancia de elmidos respecto al ensamblaje del tramo estudiado del río Jordán (Tabla 2), concuerda con Mosquera et al. (2008), quienes encontraron bajas abundancias de esta familia en ambientes acuáticos similares en el río Cali a altitudes entre los 1000 y 1 700 m.s.n.m.

Thraulodes se recolectó en todas las estaciones, según Zúñiga et al. (1997), tienen un rango de distribución altitudinal amplio, desde los 1100 m.s.n.m. en el Valle del Cauca, con una alta frecuencia de población, sin embargo, en E2 y E3 sólo se registraron 2 individuos. Estos resultados son contrastantes con estudios realizados en corrientes superficiales del noroeste del estado de Pará, Brasil (región tropical), donde la abundancia de Thraulodes fué muy baja (un individuo) (Andrade et al., 2017).

La dominancia de Rhagovelia (137 individuos) en la estación Antes Bocatoma (E2), se debió a la alteración del cauce por las obras asociadas a la bocatoma del acueducto, las cuales produjeron habitats de remanso en las orillas, adecuados para este género de patinadores. En el río Pance, que al igual que el río Jordán, vierte sus aguas al río Jamundí, Rhagovelia fue dominante en un rango de altitud similar al estudiado en el río Jordán (entre 950 y 1260 m.s.n.m.) (Departamento Administrativo de Gestión del Medio Ambiente, 1996).

Smicridea solo se encontró en la estación E2 en las zonas de rápidos, siendo un taxón escaso. En altitudes similares, Chará-Serna et al. (2010) registran Smicridea en quebradas de la región ecocafetera de Colombia en abundancias importantes.

Leptonema, género reconocido por ser indicador de buena calidad de agua, se asoció con el $\mathrm{pH}$, el cual tuvo baja variabilidad con valores cercanos al neutro, característicos de buena calidad de agua y permisibles para la vida acuática (Vásquez-Zapata 2009). Resultados similares fueron obtenidos por Bustamante-Toro, Marín-Villegas y Corredor-Coy (2011) en cuanto al comportamiento del pH en un sistema multivariado de ordenación.

En cuanto a los eventos de muestreo de cada estación, los realizados en la estación E1 se agrupan mejor con presencia de géneros sensibles a la contaminación como Anchytarsus, Psephenops y Leptohyphes. Las estaciones E2 y E3 mostraron mayor dinámica entre muestreos principalmente por las variaciones en la abundancia de Rhagovelia.

La familia Elmidae (representada en el río Jordán por los géneros Cylloepus, Hexanchorus, Heterelmis, Macrelmis y Phanocerus) es usada en programas de biomonitoreo y evaluación ambiental por la sensibilidad de la mayoría de sus especies a los cambios físicos y químicos en el ambiente (Compin \& Céréghino, 2003; Garcia-Criado \& FernandezAláez, 2001; Ribera, 2000; Ribera \& Foster, 1992), tuvo diferentes ordenamientos con las variables ambientales y los eventos de muestreo en el río Jordán (Fig. 2), posiblemente debido a que los géneros dentro de la familia 
pueden responder diferente a los cambios en la calidad del agua.

La familia Leptophlebiidae ha sido reconocida como poco tolerante a la contaminación en diferentes sistemas de bioindicación en el mundo (Alba-Tercedor \& Jimenez-Millán, 1987; Alba-Tercedor \& Sánchez-Ortega, 1988; Alba-Tercedor et al., 2002; Armitage, Moss, Wright, \& Furse, 1983; Munné, Prat, \& Cabals, 1999; Roldán-Pérez, 2003; Sanchez-Herrera, 2005; Zamora, 1999; Zamora, 2007; Zuñigade-Cardoso, Rojas-de-Hernández y Caicedo, 1993; Zuñiga-de-Cardoso \& Cardona, 2009). Si bien Thraulodes ha sido relacionado en aguas con altos niveles de oxígeno en ríos del suroccidente colombiano (Zuñiga et al., 1997), en el río Jordán este género de Leptophlebiidae se asoció negativamente con la turbidez, siendo más abundante en la estación La Estrella (E1) donde la turbidez es menor respecto a las otras estaciones.

Se puede concluir que la diversidad de macroinvertebrados acuáticos en el río Jordán disminuye a medida que aumentan los factores de estrés en la cuenca donde las variables asociadas a la degradación de la materia orgánica son las que mejor se correlacionan con los cambios espacio-temporales del ensamblaje biótico.

Declaración de ética: los autores declaran que todos están de acuerdo con esta publicación y que han hecho aportes que justifican su autoría; que no hay conflicto de interés de ningún tipo; y que han cumplido con todos los requisitos y procedimientos éticos y legales pertinentes. Todas las fuentes de financiamiento se detallan plena y claramente en la sección de agradecimientos. El respectivo documento legal firmado se encuentra en los archivos de la revista.

\section{AGRADECIMIENTOS}

A la Universidad Autónoma de Occidente por su apoyo material en laboratorios y equipos de campo. Al profesor Hilldier Zamora de la Universidad del Cauca.

\section{RESUMEN}

Debido a la influencia de diferentes actividades humanas, los ríos y su biodiversidad están bajo tensión. El objetivo de este estudio fue evaluar la diversidad de los macroinvertebrados acuáticos del río Jordán (Valle del Cauca) y su relación con variables fisicoquímicas. En el tramo evaluado $(5650 \mathrm{~m}$ ) se realizan actividades de minería, captación de agua, ganadería, agricultura, recreación y ocupación ilegal del territorio. Se ubicó una estación de muestreo a 1173 m.s.n.m. (E1), con vertimientos de agua residual doméstica. La segunda a $1069 \mathrm{~m}$ (E2) se ubicó antes de la bocatoma del acueducto, y la tercera a 1019 m (E3) después de la bocatoma. Se midieron las concentraciones de variables fisicoquímicas $(n=15)$. Los organismos fueron recolectados usando redes surber $(0.36$ $\left.\mathrm{m}^{2}, 500 \mu \mathrm{m}\right)$, de pantalla $\left(1.6 \mathrm{~m}^{2}, 350 \mu \mathrm{m}\right)$ y D-net $\left(0.9 \mathrm{~m}^{2}\right.$, $500 \mu \mathrm{m})$, evaluando la estructura del ensamblaje mediante categorías de abundancia. El ensamblaje de macroinvertebrados acuáticos se relacionó con las variables fisicoquímicas mediante un análisis de correspondencia canónica (ACC). Las variables fisicoquímicas mostraron que E3 fue la estación más contaminada. Se registraron 9 órdenes, 23 familias y 31 taxa. E3 presentó la menor riqueza y en E2 Rhagovelia fue dominante. El ACC mostró que las variables que mayor influencia tienen sobre el ensamblaje de macroinvertebrados son el oxígeno, la $\mathrm{DBO}_{5}$, los sólidos disueltos totales y la turbidez. Se concluye que la diversidad de macroinvertebrados acuáticos disminuye con el aumento de la materia orgánica posiblemente debido a los vertimientos residuales y disminución del caudal.

Palabras clave: insectos acuáticos, Elmidae, Veliidae, demanda bioquímica de oxígeno, coliformes totales, Río Jordán.

\section{REFERENCIAS}

Alba-Tercedor, J., Jáimez-Cuéllar, P., Álvarez, M., Avilés, J., Bonada, N., Casas, J., ... Zamora-Muñoz, C. (2002). Caracterización del estado ecológico de los ríos mediterráneos ibéricos mediante el índice IBMWP (antes BMWP'). Limnética, 21, 175-185.

Alba-Tercedor, J. \& Jiménez-Millán, F. (1987). Proyecto LUCDEME III: Evaluación de las variaciones estacionales de la calidad de las aguas del rio Guadalfeo, basada en el estudio de las comunidades de macroinvertebrados acuáticos. España: Instituto Nacional para la Conservación de la Naturaleza.

Alba-Tercedor, J. \& Sánchez-Ortega, A. (1988). Un método rápido y simple para evaluar la calidad biológica de las aguas corrientes basado en el de Hellawell (1978). Limnética, 4, 51-56.

Alcaldía Municipio de Jamundí. (2007). Plan Básico de Ordenamiento Territorial. Colombia: Planeación Municipal. 
Andrade, A. L., Schlemmer, L., Loureiro., N., Shimanoc, Y., Justino, A. P., Fogaça, L., ... Juen, L. (2017). Influence of oil palm monoculture on the taxonomic and functional composition of aquatic insect communities in eastern Brazilian Amazonia. Ecological Indicators, 82, 478-483.

APHA, AWWA, \& WPCF. (2005). Standard methods for the examination of waste water $\left(21^{\text {th }}\right.$ ed.). Washington D.C.: American Public Health Association.

Armitage, P. D., Moss, D., Wright, J. F., \& Furse, M. T. (1983). The performance of a new biological water quality score system based on macroinvertebrates over a wide range of unpolluted running-water sites. Water Research, 17, 333-347.

Barbour, M. T., Gerritsen, J., Snyder, B. D., \& Stribling, J. B. (1999). Rapid Bioassessment Protocols for Use in Streams and Wadeable Rivers: Periphyton, Benthic Macroinvertebrates and Fish ( $2^{\text {nd }}$ ed.). Washington D.C.: Environmental Protection Agency - EPA.

Beauger, A., Lair, N., Reyes-Marchant, P., \& Peiry, J. L. (2006). The distribution of macroinvertebrate assemblages in a reach of the River Allier (France), in relation to riverbed characteristics. Hydrobiologia, $571,63-76$

Berg, K. (1948). Biological studies on the River Sousaa. Folia Limnologica Scandinavica, 4, 1-318.

Bohórquez, H., Reinoso, G., \& Guevara, G. (2011). Seasonal size distribution of Anacroneuria (Plecoptera: Perlidae) in an Andean tropical river. Revista Colombiana de Entomología, 2, 305-312.

Bustamante-Toro, C. A., Marín-Villegas, N. C., \& Corredor-Coy, N. V. (2011). Estudio de calidad ambiental en la quebrada La Florida, unidad de manejo de cuenca del río Quindío. Armenia - Quindío, Colombia. Revista de la Asociación Colombiana de Ciencias Biológicas, 23, 65-76.

Camargo, J. A., Alonso, A., \& de la Puente, M. (2005). Eutrophication downstream from small reservoirs in mountain rivers of Central Spain. Water Research, 39, 3376-3384.

Castro, M. I. \& Donato, J. C. (2008). Aspectos generales sobre la ecología de ríos. Ecología de un río de montaña de los andes colombianos (Río Tota, Boyacá). Bogotá D.C.: Universidad Nacional de Colombia.

Chacón, M. M. \& Segnini, S. (1996). Reconocimiento taxonómico de las náyades del orden ephemeroptera en la deriva de dos ríos de alta montaña en el estado de Mérida, Venezuela. Boletín de Entomología Venezolana, 2, 103-122.

Chará-Serna, A. M., Chará, J. D., Zúñiga, M. C., Pedraza, G. X., \& Giraldo, L. P. (2010). Clasificación trófica de insectos acuáticos en ocho quebradas protegidas de la ecorregión cafetera colombiana. Universitas Scientiarum, 15, 27-36.

Clarke, K. R., \& Gorley, R. N. (2015). PRIMER Plymouth Routines In Multivariate Ecological Research (version 7). Retrieved from https://www.primer-e.com

Colwell, R. K. (2006). EstimateS Statistical estimation of species richness and shared species from samples (version 8). Retrieved from http://viceroy.eeb.uconn. edu/estimates

Compin, A., \& Céréghino, R. (2003). Sensitivity of aquatic insect species richness to disturbance in the Adour - Garonne stream system (France). Ecological Indicators, 3, 135-142.

Corporación Autónoma Regional del Valle del Cauca CVC. (2009). Conjunto de aplicativos sistematizados para divulgación y análisis de la información del recurso hídrico en el Valle del Cauca. Colombia: CVC.

Corporación Autónoma Regional del Valle del Cauca CVC, DAGMA, \& Parques Nacionales Naturales de Colombia. (2007). Plan de Ordenación y Manejo de Cuenca Hidrográfica del río Jamundi. Colombia: CVC.

Covich, A. P. (1988). Geographical and historical comparison of Neotropical stream: biotic diversity and detrital procesing in highly variable habitat. Journal of the North American Benthological Society, 7, 361-386.

De-Castellanos, Z. (1994). Los ephemeroptera en Argentina. Fauna de agua dulce de la República Argentina, La Plata (Vol. 33). Argentina: Programa de Fauna de Agua Dulce.

Decreto No 1594. (1984). Diario Oficial de la República de Colombia, Bogotá, Colombia, 23 de julio de 1984.

Dominguez, E., \& Fernandez, H. R. (2009). Macroinvertebrados bentónicos sudamericanos: Sistemática y biología. Argentina: Fundación Miguel Lillo.

Domínguez, E., Molineri, C., Pescador, M. L., Hubbard, M. D., \& Nieto C. (2006). Ephemeroptera of South America. In J. Adis, J. R. Arias, G. Rueda-Delgado, \& K. M. Wantzen (Eds.), Aquatic Biodiversity in Latin America (ABLA) (Vol. 2). Moscow-Sofia: Pensoft.

Fernández, H., Romero, F., Peralta, M., \& Grosso, L. (2001). La diversidad del zoobentos en ríos de montaña del noroeste de Argentina: comparación entre seis ríos. Ecología Austral, 11, 9-16.

Fundación para la vida en comunidad vivir - Funvivir. (2008). Plan de Conservación y Restauración de la subcuenca del río Jamundi. Colombia: Corporación Autónoma Regional del valle del Cauca - CVC.

García, P. J., Cantera, J., Zuñiga, M. C., \& Montoya, J. (2009). Estructura y diversidad de las comunidades 
de macroinvertebrados acuáticos en la cuenca baja del río Dagua (Andén Pacífico Vallecaucano-Colombia). Revista Asociación Colombiana de Ciencias Biológicas, 13, 25-48.

Garcia-Criado, F., \& Fernandez-Aláez, M. (2001). Hydraenidae and Elmidae assemblages (Coleoptera) from a Spanish river basin: good indicators of coal mining pollution? Archiv fur Hydrobiologie, 150, 641-660.

Gotelli, N. J., \& Colwell, R. K. (2011). Estimating species richness. 2011. In A. E. Magurren \& B. J. McGill (Eds.), Biological diversity frontiers in measurement and assessment (pp. 39-54). Oxford: University Press.

Hamada, N., Thorp, J. H., \& Rogers, D. C. (2018). Thorp and Covich's Freshwater Invertebrates (Vol. 3). Keys to Neotropical Hexapoda. UK: Academic Press.

Jáimez-Cuellar, P., Vivas, S., Bonada, N., Robles, S., Mellado, A., Alvarez, M., ... Alba-Tercedor, J. (2002). Protocolo GUADALMED (PRECE). Limnetica, 3, 187-204

Januschke, K., Jähnig, S., Lorenz, A., \& Herin, D. (2014). Mountain river restoration measures and their success(ion): Effects on river morphology, local species pool, and functional composition of three organism groups. Ecological Indicators, 38, 243-255.

Klemm, D. J., Lewis, P. A., Fulk, F., \& Lazorchak, J. M. (1990). Macroinvertebrate field and laboratory methods for evaluating the biological integrity of surface waters. EPA/600/4-90/030. USA: US Environmental Protection Agency.

Kobayasi, H., \& Ando, K. (1975). Diatoms from HozojiNuma, Jizoin-Numa and Nakashinden-Numa ponds in Hanyu City, Saitama Prefecture. Bulletin of Tokyo Gakugei University, 27, 178-204.

Lobo, E. A. \& Leighton, G. (1986). Estructuras comunitarias de las fitocenosis planctónicas de los sistemas de desembocaduras de ríos y esteros de la zona central de chile. Revista de Biología Marina, 22, 1-29.

Machado-Cartagena, T. (1989). Distribución ecológica e identificación de los coleópteros acuáticos en diferentes pisos altitudinales del departamento de Antioquia. Colombia: Universidad de Antioquia.

Margalef, R. (1983). Limnología. Barcelona: Omega.

Merrit, R. W., Cummins, K. W., \& Berg, M. B. (2008). An introduction to the aquatic insects of north America. Dubuque: Hunt Publishing Company.

Mosquera-Restrepo, D., Palacios-Peñaranda, M. L., \& Soto-Duque, A. (2008). Bioindicación de la calidad del agua del río Cali, Valle del Cauca, Colombia, usando macroinvertebrados acuáticos. Revista Asociación Colombiana de Ciencias Biológicas, 43, 130-143.
Munné., A., Prat, N., \& Cabals, I. (1999). Qualitat biològica del riu Anoia: diagnosi de l'estat del riu i dels trams finals dels afluents principals. Estudis de la qualitat ecològica dels rius. Barcelona: Área de medi ambient de la diputació.

Muñoz, F. (1996). Manual de trichoptera neotropical, anotaciones y claves de las familias y géneros conocidos de trichoptera para centro y sur América. Minnesota: Departamento de Entomología Universidad de Minnesota.

Ottoboni-Segura, M., Valente-Neto, F., \& Fonseca-Gessner, A. (2011). Elmidae (Coleoptera, Byrrhoidea) larvae in the state of São Paulo, Brazil: Identification key, new records and distribution. Zookeys, 151, 53-74.

Palmer, M. W. (1993). Putting things in even better order: The advantages of canonical correspondence analysis. Ecology, 22, 15-30.

Posada-García, J. A. \& Roldán-Pérez, G. (2003). Clave ilustrada y diversidad de las larvas de trichoptera en el nor-occidente de Colombia. Caldasia, 25, 169-192.

Ramírez, A., Restrepo, R., \& Viña-Viscaíno, G. (1997). Cuatro índices de contaminación para caracterización de aguas continentales: formulaciones y aplicaciones. Ciencia, Tecnología y Futuro, 1, 135-153.

Ribera, I. (2000). Biogeography and conservation of Iberian water beetles. Biological Conservation, 92, 131-150.

Ribera, I. \& Foster, G. N. (1992). Uso de coleópteros acuáticos como indicadores biológicos (coleoptera). Elytron, 6, 61-75.

Richards, C., Haro, R. J., Johnson, L. C., \& Host, G. E. (1997). Catchment and reach-scale properties as indicators of macroinvertebrate species traits. Freshwater Biology, 37, 219-230.

Robinson, C. T., Tockner, K., \& Ward, J. V. (2002). The fauna of dynamic riverine landscapes. Freshwater Biology, 47, 661-677.

Roldán-Pérez, G. (2003). Bioindicación de la calidad del agua en Colombia. Uso del método BMWP/col. Medellín: Editorial Universidad de Antioquia.

Roldán-Pérez, G. \& Ramirez-Restrepo, J. (2008). Fundamentos de limnología neotropical. Medellín: Universidad de Antioquia.

Rosenberg, D. M., \& Resh, V. H. (1993). Introduction to Freshwater Biomonitoring and Benthic Macroinvertebrates. In D. M. Rosenberg \& V. H. Resh (Eds.), Freshwater Biomonitoring and Benthic Macroinvertebrates (pp. 1-9). New York: Chapman/Hall.

Rosguen, D. L. (1994). A classification for natural rivers. Catena, 22, 169-199. 
Sánchez-Herrera, M. J. (2005). El índice biológico BMWP, modificado y adaptado al cauce principal de río Pamplonita Norte de Santander. Revista de la Facultad de Ciencias Básicas Universidad de Pamplona, 3, 54-67.

Sánchez-Vélez, A. \& García-Núñez, R. M. (1999). Biomonitoreo de ríos en la gestión de cuencas; una aproximación introductoria. Culiacán, Sinaloa, México. IX Congreso Nacional de Irrigación. Simposio 4 Manejo Integral de Cuencas Hidrológicas. México.

Silva-Passos, M. I., Nessimian, J. L., \& Ferreira, N. (2007). Chaves para identificação dos géneros de elmidae (coleoptera) ocorrentes no estado do Río de Janeiro, Brasil. Revista Brasileira de Entomología, 51, 42-53.

Thorp, J. H., \& Rogers, D. C. (2015). Thorp and Covich's Freshwater Invertebrates, Ecology and General Biology. UK: Academic Press.

Vásquez-Zapata, G. L. (2009). Calidad de las aguas naturales en relación con el régimen de caudal ambiental. En J. R. Cantera-Kintz, Y. Carvajal-Escobar, \& L. M. Castro-Heredia (Eds.), Caudal ambiental: conceptos, experiencias y desafios (pp. 137-166). Cali: Programa Editorial Universidad del Valle.

Villarreal, H., Álvarez, M., Córdoba, S., Escobar, F., Fagua, G., Gast, F., ... Umaña, A. M. (2006). Manual de métodos para el desarrollo de inventarios de biodiversidad. Bogotá: Instituto de Investigación de Recursos Biológicos Alexander Von Humboldt.

Wyzga, B., Amirowicz, A., Paweł, O., Hajdukiewicz, H., Radecki-Pawlikc, A., Zawiejskad, J., \& Mikus, P. (2014). Response of fish and benthic invertebrate communities to constrained channel conditions in a mountain river: Case study of the Biała, Polish Carpathians. Limnologica, 46, 58-69.
Zamora, H. (1999). Adaptación del índice BMWP para la evaluación biológica de la calidad de las aguas epicontinentales en Colombia. Unicauca Ciencia, 4, 47-59.

Zamora, H. (2007). El índice BMWP y la evaluación biológica de la calidad del agua en los ecosistemas acuáticos epicontinentales naturales de Colombia. Revista de la Asociación Colombiana de Ciencias Biológicas, 19, 73-81.

Zuñiga-de-Cardoso, M. C. (2010). Diversidad, distribución y ecología del orden plecoptera (insecta) en Colombia, con énfasis en Anacroneuria (Perlidae). Momentos de Ciencia, 7, 101-112.

Zuñiga-de-Cardoso, M. C. \& Cardona, W. (2009). Bioindicadores de calidad de agua y caudal ambiental. En J. R. Cantera-Kintz, Y. Carvajal-Escobar, \& L. M. Castro-Heredia (Eds.), Caudal ambiental: conceptos, experiencias y desafios (pp. 303-310). Cali: Programa Editorial Universidad del Valle.

Zuñiga-de-Cardoso, M. C., Rojas-de-Hernández, A. M., \& Caicedo, G. (1993). Indicadores ambientales de calidad de agua en la cuenca del Río Cauca. Revista Asociación de Ingenieros Sanitarios de Antioquia AINSA, 13, 17-27.

Zuñiga-de-Cardoso, M. C., Rojas-de-Hernández, A. M., \& Mosquera-de-Aguilera, S. (1997). Biological aspects of ephemeroptera in rivers of southwestern Colombia (South América). Ephemeroptera \& Plecoptera: Biology-Ecology-Systematics. En P. Landolt \& M. Sartori (Eds.), Ephemeroptera \& Plecoptera. Biology-Ecology-Systematics (pp. 261-268). Fribourg: Mauron + Tinguely \& Lachat, S.A. 